\title{
A TOPOLOGIA MOLECULAR QTAIM E A DESCRIÇÃO MECÂNICO-QUÂNTICA DE LIGAÇÕES DE HIDROGÊNIO E LIGAÇÕES DE DI-HIDROGÊNIO
}

\author{
Boaz G. Oliveira* e Regiane C. M. U. Araújo \\ Departamento de Química, Universidade Federal da Paraíba, 58059-900 João Pessoa - PB, Brasil \\ Mozart N. Ramos \\ Departamento de Química Fundamental, Universidade Federal de Pernambuco, 50739-901 Recife - PE, Brasil
}

Recebido em 26/6/09; aceito em 10/1/10; publicado na web em 23/4/10

\begin{abstract}
THE QTAIM MOLECULAR TOPOLOGY AND THE QUANTUM-MECHANICAL DESCRIPTION OF HYDROGEN BONDS AND DIHYDROGEN BONDS. Hydrogen bonds formed through the interaction between a high electronic density center (lone electron pairs, $\pi$ or pseudo- $\pi$ bonds) and proton donors cause important electronic and vibrational phenomena in many systems. However, it was demonstrated that proton donors interact with hydrides, such as alkali and alkaline earth metals $\left(\mathrm{BeH}_{2}, \mathrm{MgH}_{2}, \mathrm{LiH}\right.$ and $\mathrm{NaH}$ ), what yields a new type of interaction so-called dihydrogen bonds. The characterization of these interactions has been performed at light of the Quantum Theory of Atoms in Molecules (QTAIM), by which the electronic densities $\rho$ are quantified and the intermolecular regions are characterized as closed-shell interactions through the analysis of the Laplacian field $\nabla^{2} \rho$.
\end{abstract}

Keywords: QTAIM; hydrogen bonds; dihydrogen bonds.

\section{INTRODUÇÃO}

Após publicar alguns trabalhos sobre a natureza da ligação química, ${ }^{1}$ em 1939 Pauling editou o clássico livro texto "Nature of the Chemical Bond and the Structure of Molecules and Crystals: An Introduction to Modern Structural Chemistry". ${ }^{2}$ Este livro é considerado uma das publicações mais importantes da história da química, cuja leitura tem auxiliado experimentais e teóricos no estudo de diversos fenômenos como, por exemplo, a hibridização em órbitas atômicas e a natureza planar das ligações peptídicas. Segundo Pauling, se a distância entre dois átomos interagentes for menor ou pelo menos igual à soma dos raios atômicos de van der Waals, ${ }^{3}$ pode-se afirmar que estes átomos estão quimicamente ligados. ${ }^{4}$ Desde então, este critério se tornou bastante respeitado, sendo vastamente utilizado como justificativa na formação de interações intermoleculares nos mais variados sistemas ${ }^{5}$ como, por exemplo, os complexos de hidrogênio. ${ }^{6,7}$ Algumas décadas após Pauling ter publicado suas idéias sobre ligação química, o grupo liderado por J. A. Pople propôs os primeiros conjuntos de base $a b$ initio, os quais foram desenvolvidos com o objetivo de descrever o orbital molecular através da implementação de formulações matemáticas em pacotes computacionais. ${ }^{8}{ }^{89}$ Estes métodos computacionais provocaram uma verdadeira revolução na pesquisa científica, ${ }^{10} \mathrm{e}$ atualmente são utilizados por muitas entidades acadêmicas e centros industriais.

Contemporâneo de Pople, R. F. W. Bader também criou um método para estudar a estrutura eletrônica e, principalmente, a ligação química. ${ }^{11}$ Batizada por Bader de Teoria Quântica de Átomos em Moléculas (QTAIM - Quantum Theory of Atoms in Molecules), ${ }^{12}$ este modelo quântico é considerado inovador no estudo da ligação química, ${ }^{13}$ mas também eficiente na caracterização de interações intra e/ou intermoleculares. ${ }^{14,15}$ Revivendo alguns conceitos, sabe-se que toda e qualquer informação relevante a um sistema é contida em uma função de onda $\psi$, pela qual o valor de uma propriedade física é obtido por intermédio da ação de um operador matemático como, por exemplo, o Hamiltoniano total. ${ }^{16}$ Segundo os conceitos mecânicos-quânticos da QTAIM, ${ }^{17,18}$ as propriedades observáveis

*e-mail: boazgaldino@gmail.com de um sistema químico estão contidas em sua densidade eletrônica molecular, $\rho$. Mesmo sendo uma das primeiras observáveis usadas na ciência ${ }^{19,20}$ como, por exemplo, na implementação da Teoria do Funcional de Densidade (DFT - Density Functional Theory) ${ }^{21,22}$ por Hohenberg, Kohn e Sham, ${ }^{23,24}$ a densidade eletrônica é também considerada um parâmetro físico-químico bastante polêmico devido à inexistência de uma expressão analítica clara que a descreva e que possa ser usada na interpretação da concentração de carga em sítios moleculares específicos. Trabalhando neste sentido, alguns cientistas têm apresentado algumas propostas como, por exemplo, a baseada nos conceitos de Hirshfeld ${ }^{25,26}$ descrita pela Equação 1.

$$
\mathrm{I}=f \rho \mathrm{A}(\mathrm{r}) \ln \frac{\rho A_{(\mathrm{r})}}{\rho \mathrm{A}^{\mathrm{o}}(\mathrm{r})} \mathrm{dr}+f \rho \mathrm{B}(\mathrm{r}) \ln \frac{\rho \mathrm{B}(\mathrm{r})}{\rho \mathrm{B}^{\mathrm{o}}(\mathrm{r})} \mathrm{dr}
$$

Nesta expressão, I define uma quantidade de carga eletrônica perdida na sobreposição atômica, ou seja, uma diferença entre as densidades eletrônicas dos átomos A e B livres $\left(\rho \mathrm{A}_{(\mathrm{r})}^{\circ} \mathrm{e} \mathrm{B}^{\circ}{ }_{(\mathrm{r})}\right)$ e quando formam a ligação química $\mathrm{A}-\mathrm{B}\left(\rho \mathrm{A}_{(\mathrm{r})}\right.$ e $\left.\rho \mathrm{B}_{(\mathrm{r})}\right)$. Na abordagem da QTAIM, a densidade eletrônica é usada como observável mecânico-quântico para execução de integrações numéricas, onde o vetor gradiente $\nabla \rho$ é a condição básica para se determinar a topologia molecular. ${ }^{27}$ Considerando os átomos como sistemas de camada aberta $(\Omega)$ e restringindo condições de contorno na superfície molecular $\mathrm{S}_{(\Omega, \mathrm{r})}$, Bader utilizou destes argumentos para mostrar que o fluxo de densidade de carga é nulo em qualquer ponto desta superfície. ${ }^{28}$ Como consequência, o gradiente $\nabla \rho$ é perpendicular a um vetor unitário $\mathrm{n}_{(\mathrm{r})}$ normal à superfície definida em $\mathrm{S}_{(\Omega, \mathrm{r})},{ }^{29}$ conforme demonstrado pela Equação 2:

$$
\nabla \rho . \mathrm{n}_{(\mathrm{r})}=0
$$

A partir de uma sequência de vetores gradientes $\nabla \rho$ é que são obtidas todas as trajetórias ou linhas de contorno da densidade eletrônica, as quais podem ser ascendentes ou em declive. Na prática, as trajetórias de $\nabla \rho$ são bem definidas por um ponto específico no espaço, denominado de atrator. ${ }^{30}$ Como o gradiente da densidade eletrônica é função de seus atratores, estes são propriamente os núcleos do sistema molecular. Considerando todos os atratores nucleares, o conjunto de 
trajetórias do gradiente forma uma base atômica ou o próprio sistema de camada aberta $\Omega$. Em termos de QTAIM, o termo $\Omega$ recebe uma definição químico-quântica traduzida simplesmente como "átomo". ${ }^{31}$

Não restrita apenas ao ambiente atômico, a QTAIM também trata a formação da ligação química. ${ }^{32}$ Conforme as trajetórias do gradiente da densidade eletrônica se moldam para descrever um sistema químico, estas trajetórias não se originam do infinito, mas de um ponto localizado entre dois atratores, os chamados Pontos Críticos de Ligação (BCP Bond Critical Point).$^{33} \mathrm{~A}$ identificação dos BCP foi apresentada como uma proposta evolutiva aos conceitos da população de recobrimento equivalente de Mulliken, ${ }^{34}$ a qual foi usada durante muito tempo em estudos de ligações químicas e interações intermoleculares. ${ }^{35}$ Quando duas trajetórias de $\nabla \rho$ direcionadas aos núcleos são formadas a partir de um único BCP, estas são chamadas de Linhas Interatômicas (IL Interatomic Lines) ${ }^{36}$ ou Trajetórias de Ligação (BP - Bond Path) ${ }^{37} \mathrm{Em}$ outras palavras, quando algum BP interliga dois atratores, admite-se que estes dois núcleos formam uma ligação química. ${ }^{38} \mathrm{~A}$ localização dos BCP é feita através do Laplaciano da densidade eletrônica $\left(\nabla^{2} \rho\right)$ obtido conforme a Equação 3, onde os três autovalores $\left(\lambda_{1}, \lambda_{2}\right.$ e $\left.\lambda_{3}\right)$ de $\rho$ possuem dois valores negativos $\left(\lambda_{1}\right.$ e $\left.\lambda_{2}\right)$ e um positivo $\left(\lambda_{3}\right)$.

$$
{ }^{2} \rho=\frac{\delta^{2} \rho}{\delta_{\mathrm{X}}^{2}}+\frac{\delta^{2} \rho}{\delta_{\mathrm{y}}^{2}}+\frac{\delta^{2} \rho}{\delta_{\mathrm{Z}}^{2}} \quad \lambda_{1}+\lambda_{2}+\lambda_{3}
$$

Estas três derivadas de $\rho$ são obtidas a partir da diagonalização de uma matriz Hessiana simétrica ( 3 x 3). Esta diagonalização representa uma rotação das coordenadas do sistema molecular mediante uma sobreposição com os eixos de curvatura (x, y e z) do BCP, e é neste ponto onde o Laplaciano é calculado para ser utilizado como parâmetro na modelagem topológica da ligação química. ${ }^{39}$ Como exemplo, o somatório dos três autovalores de $\lambda_{1}, \lambda_{2}$ e $\lambda_{3}$ fornece o resultado $(-1)+(-1)+(+1)=-1$, que representa o BCP como $(3,-1)$. Além do $\mathrm{BCP}$, os autovalores da matriz Hessiana podem fornecer outros pontos críticos de mínimos e máximos, tais como os Atratores Nucleares (NA - Nuclear Attractor) ${ }^{40}$ e os Pontos Críticos de Anel (RCP - Ring Critical Points), ${ }^{41}$ os quais são definidos como $(3,-3)$ e $(3,+1)$, respectivamente. Outra informação importante está na relação entre o Laplaciano $\nabla^{2} \rho$ num ponto (r) e a elipicidade ( $(\varepsilon)$, a qual é definida como $\varepsilon=\lambda_{1} / \lambda_{2}-1$, que é um valor positivo sempre que $\lambda_{1}<$ $\lambda_{2}<0$ e $\lambda_{3}>0 .^{42} \mathrm{~A}$ elipicidade é um parâmetro usado para descrever o caráter da ligação química, seja covalente ou insaturada. ${ }^{43}$ Analisando a Figura 1, observa-se que $\lambda_{1}$ e $\lambda_{2}$ estão orientados em um plano perpendicular ao eixo internuclear dos átomos $\mathrm{A}$ e $\mathrm{B}$. Considerando que o valor de $\lambda_{1}$ é muito alto, temos um caráter de ligação $\pi$, enquanto que $\lambda_{1}=\lambda_{2}$ indica um formato "cilíndrico" simbolizando uma ligação sigma ou covalente. ${ }^{43}$ Este é um dos critérios da QTAIM usados para determinar a estabilidade molecular, ressonância e força interatômica.

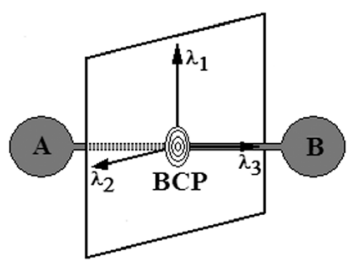

Figura 1. Ilustração esquemática do ponto crítico de ligação $(B C P)$ entre dois átomos $A$ e $B$ e a orientação tridimensional $(x, y, z)$ das elipicidades $\lambda_{1}, \lambda_{2} e \lambda_{3}$

Todavia, os campos Laplacianos, ou as segundas derivadas da densidade eletrônica trazem informações vitais para a compreensão do sistema químico. ${ }^{44} \mathrm{O}$ sinal do Laplaciano $\nabla^{2} \rho$ tem a propriedade de descrever a concentração de densidade eletrônica em uma determinada zona de ligação. ${ }^{45}$ Por exemplo, a densidade eletrônica se acumula nos BCP das ligações químicas se $\nabla^{2} \rho<0$. Por outro lado, $\nabla^{2} \rho>0$ indica que os núcleos atômicos suportam toda a concentração de carga ${ }^{46}$ Conforme a Equação 4, esta interpretação do Laplaciano é justificada pelo teorema virial da densidade eletrônica total, ${ }^{47}$ pelo qual podemos afirmar que as energias cinética $(\mathrm{K})$ e potencial $(\mathrm{U})$ se relacionam, respectivamente, com depressões e concentrações de densidade eletrônica. ${ }^{48}$ Ou seja, $\nabla^{2} \rho>0(\mathrm{~K})$ caracteriza interações intra ou intermoleculares (ligações de hidrogênio e ligações de di-hidrogênio), ${ }^{49}$ enquanto $\nabla^{2} \rho<0$ (U) descreve as ligações covalentes ou insaturadas (hidrocarbonetos ou sistemas biológicos hiperconjugados). ${ }^{50,51}$

$$
\nabla^{2} \rho=2 \mathrm{~K}+\mathrm{U}
$$

Através da densidade eletrônica $\rho$, do Laplaciano $\nabla^{2} \rho$, e da elipicidade $\varepsilon$, muitos estudos têm sido realizados a fim de elucidar fenômenos químicos fundamentais como, por exemplo, o comportamento ácido/base segundo a teoria de Lewis. ${ }^{52}$ Resumidamente, QTAIM proporciona uma descrição quântica sobre a localização e comportamento dos átomos em uma ligação química. ${ }^{53,54}$

\section{COMPLEXOS DE HIDROGÊNIO П, NÃO-USUAL П E PSEUDO-П}

Baseado em um rigoroso critério formulado por Koch e Popelier, ${ }^{55}$ sistemas $\pi$ e p- $\pi$ formados por ligação de hidrogênio ${ }^{56}$ podem ser estudados através de parâmetros topológicos derivados da análise da densidade eletrônica molecular. ${ }^{57}$ A partir dos conceitos da QTAIM, BCP são localizados em ligações de hidrogênio formadas por doadores de próton e elétrons $\pi{ }^{58,59}$ Para os complexos $\pi \mathrm{C}_{2} \mathrm{H}_{2} \cdots 2 \mathrm{HF}(\mathbf{a})$, nu- $\pi \mathrm{C}_{2} \mathrm{H}_{4} \cdots 2 \mathrm{HF}$ (b) e p- $\pi \mathrm{C}_{3} \mathrm{H}_{6} \cdots 2 \mathrm{HF}$ (c), ilustrados na Figura 2, os valores dos parâmetros topológicos $\rho$ e $\nabla^{2} \rho$ estão, respectivamente, listados nas Tabelas 1 e $2 .{ }^{60} \mathrm{O}$ complexo $\mathrm{C}_{2} \mathrm{H}_{4} \cdots 2 \mathrm{HF}$ é denominado como $\pi$ porque a ligação de hidrogênio ou mais especificamente o ácido fluorídrico (HF) interage exatamente no centro da ligação $\pi(\mathrm{C} \equiv \mathrm{C})$ do etileno $\left(\mathrm{C}_{2} \mathrm{H}_{4}\right)$. No caso do complexo $\mathrm{C}_{2} \mathrm{H}_{2} \cdots 2 \mathrm{HF}$, este se caracteriza por uma ligação de hidrogênio $\pi$ não-usual (nu) pois a primeira espécie ácida não interage com a ligação $\pi$, mas com um dos carbonos acetilênicos, conforme ilustrado pelos BCP da Figura $3 .{ }^{61}$ Por fim, o complexo homocíclico $\mathrm{C}_{3} \mathrm{H}_{6} \cdots 2 \mathrm{HF}$ tem como fonte doadora de elétrons a ligação $(\mathrm{C}-\mathrm{C})$, a qual é considerada uma ligação pseudo $(\mathrm{p}) \pi$, devido à tensão do anel ciclopropano $\left(\mathrm{C}_{3} \mathrm{H}_{6}\right)$.

De acordo com os valores negativos do Laplaciano, verifica-se uma concentração de carga eletrônica nas ligações $(\mathrm{C}-\mathrm{C}),(\mathrm{C}=\mathrm{C})$, $\mathrm{C} \equiv \mathrm{C})$ e $(\mathrm{H}-\mathrm{F})$. Entretanto, as densidades eletrônicas nas ligações $(\mathrm{C}-\mathrm{C}),(\mathrm{C}=\mathrm{C})$ e $\mathrm{C} \equiv \mathrm{C})$ são praticamente invariáveis se compararmos cada tipo de complexo de hidrogênio bi e trimolecular, como pode ser observado nos valores de 0,411 e 0,410 e/a ${ }_{0}^{3}$ para $\mathrm{C}_{2} \mathrm{H}_{2} \cdots \mathrm{HF}$ e $\mathrm{C}_{2} \mathrm{H}_{2} \cdots 2 \mathrm{HF} ; 0,342$ e $0,343 \mathrm{e}^{-} \mathrm{a}_{0}{ }^{3}$ para $\mathrm{C}_{2} \mathrm{H}_{4} \cdots \mathrm{HF}$ e C $\mathrm{H}_{4} \cdots 2 \mathrm{HF} ;$ e 0,222 e $0,218 \mathrm{e}^{-} \mathrm{a}_{0}{ }^{3}$ para $\mathrm{C}_{3} \mathrm{H}_{6} \cdots \mathrm{HF}$ e $\mathrm{C}_{3} \mathrm{H}_{6} \cdots 2 \mathrm{HF}$, respectivamente. Por outro lado, constatamos que a densidade eletrônica de $0,369 \mathrm{e} / \mathrm{a}_{0}^{3}$ na ligação

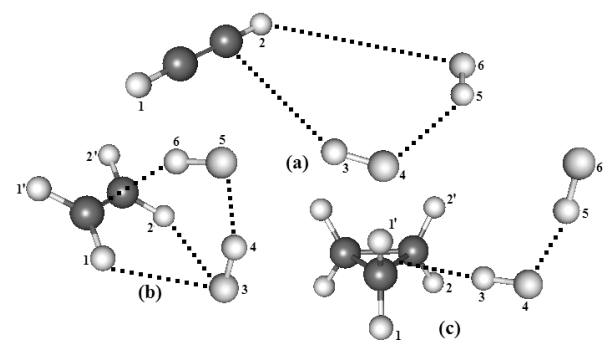

Figura 2. Geometrias otimizadas dos complexos de hidrogênio trimoleculares nu- $\pi \mathrm{C}_{2} \mathrm{H}_{2} \cdots 2 \mathrm{HF}(\boldsymbol{a}), \pi \mathrm{C}_{2} \mathrm{H}_{4} \cdots 2 \mathrm{HF}(\boldsymbol{b})$ e $p-\pi \mathrm{C}_{3} \mathrm{H}_{6} \cdots 2 \mathrm{HF}$ (c) utilizando o cálculo $B 3 L Y P / 6-311++G(d, p)$ 
Tabela 1. Valores das densidades eletrônicas ( $\rho$ em e/a ${ }_{0}{ }^{3}$ ) nos BCP dos complexos de hidrogênio $\pi \mathrm{C}_{2} \mathrm{H}_{2} \cdots \mathrm{HF}$, nu- $\pi \mathrm{C}_{2} \mathrm{H}_{2} \cdots 2 \mathrm{HF}, \pi \mathrm{C}_{2} \mathrm{H}_{4} \cdots \mathrm{HF}, \pi \mathrm{C}_{2} \mathrm{H}_{4} \cdots 2 \mathrm{HF}$, p- $\pi \mathrm{C}_{3} \mathrm{H}_{6} \cdots \mathrm{HF}$ e p- $\pi \mathrm{C}_{3} \mathrm{H}_{6} \cdots 2 \mathrm{HF}$

\begin{tabular}{|c|c|c|c|c|c|c|}
\hline \multirow{2}{*}{ Densidades } & \multicolumn{6}{|c|}{ Complexos de hidrogênio } \\
\hline & $\mathrm{C}_{2} \mathrm{H}_{2} \cdots \mathrm{HF}$ & $\mathrm{C}_{2} \mathrm{H}_{2} \cdots 2 \mathrm{HF}$ & $\mathrm{C}_{2} \mathrm{H}_{4} \cdots \mathrm{HF}$ & $\mathrm{C}_{2} \mathrm{H}_{4} \cdots 2 \mathrm{HF}$ & $\mathrm{C}_{3} \mathrm{H}_{6} \cdots \mathrm{HF}$ & $\mathrm{C}_{3} \mathrm{H}_{6} \cdots 2 \mathrm{HF}$ \\
\hline$\overline{\rho_{(\mathrm{H}}{ }^{3}-{ }_{\mathrm{F})}^{4}}$ & 0,354 & 0,339 & 0,353 & 0,336 & 0,355 & 0,339 \\
\hline$\rho_{(\mathrm{H}}^{5}-_{\mathrm{F})}^{6}$ & - & 0,349 & - & 0,345 & - & 0,352 \\
\hline$\rho_{(\mathrm{C}=\mathrm{C})}$ & 0,411 & 0,410 & - & - & - & - \\
\hline$\rho_{(\mathrm{C}=\mathrm{C})}$ & - & - & 0,342 & 0,343 & - & - \\
\hline$\rho_{(\mathrm{C}-\mathrm{C})}$ & - & - & - & - & 0,222 & 0,218 \\
\hline$\rho_{(\pi \cdots \mathrm{H})}$ & 0,018 & - & 0,017 & 0,024 & - & - \\
\hline$\rho_{(\mathrm{nu}-\pi \cdots \mathrm{H})}$ & - & 0,023 & - & - & - & - \\
\hline$\rho_{(\mathrm{p}-\pi \cdots H)}$ & - & - & - & - & 0,023 & 0,024 \\
\hline$\rho_{\left(\mathrm{F}^{4} \cdots \mathrm{H}^{5}\right)}$ & - & 0,029 & - & 0,040 & - & 0,030 \\
\hline$\rho_{\left(\mathrm{F}^{6} \cdots \mathrm{H}^{2}\right)}$ & - & 0,007 & - & - & - & - \\
\hline$\rho_{\left(\mathrm{F}^{3} \cdots \mathrm{H}^{2}\right)}$ & - & - & - & 0,021 & - & - \\
\hline
\end{tabular}

*Valor de $\rho$ no ácido fluorídrico livre é $0,369 \mathrm{e} / \mathrm{a}_{0}{ }^{3}$ * Valores de $\rho_{(\mathrm{C}=\mathrm{C})}, \rho_{(\mathrm{C}=\mathrm{C})}$ e $\rho_{(\mathrm{C}-\mathrm{C})}$ nos monômeros $\mathrm{C}_{2} \mathrm{H}_{2}, \mathrm{C}_{2} \mathrm{H}_{4}$ e $\mathrm{C}_{3} \mathrm{H}_{6}$ são 0,$411 ; 0,344$ e 0,234 e/a ${ }_{0}{ }^{3}$, respectivamente.

Tabela 2. Valores dos Laplacianos da densidade eletrônica $\left(\nabla^{2} \rho\right.$ em e/a ${ }_{0}^{5}$ ) nos BCP dos complexos de hidrogênio $\pi \mathrm{C}_{2} \mathrm{H}_{4} \cdots \mathrm{HF}, \pi \mathrm{C}_{2} \mathrm{H}_{4} \cdots 2 \mathrm{HF}, \pi \mathrm{C}_{2} \mathrm{H}_{2} \cdots \mathrm{HF}$, nu- $\pi \mathrm{C}_{2} \mathrm{H}_{2} \cdots 2 \mathrm{HF}$, p- $\pi \mathrm{C}_{3} \mathrm{H}_{6} \cdots \mathrm{HF}$ e p- $\pi \mathrm{C}_{3} \mathrm{H}_{6} \cdots 2 \mathrm{HF}$

\begin{tabular}{|c|c|c|c|c|c|c|}
\hline \multirow{2}{*}{ Laplacianos } & \multicolumn{6}{|c|}{ Complexos de hidrogênio } \\
\hline & $\mathrm{C}_{2} \mathrm{H}_{2} \cdots \mathrm{HF}$ & $\mathrm{C}_{2} \mathrm{H}_{2} \cdots 2 \mathrm{HF}$ & $\mathrm{C}_{2} \mathrm{H}_{4} \cdots \mathrm{HF}$ & $\mathrm{C}_{2} \mathrm{H}_{4} \cdots 2 \mathrm{HF}$ & $\mathrm{C}_{3} \mathrm{H}_{6} \cdots \mathrm{HF}$ & $\mathrm{C}_{3} \mathrm{H}_{6} \cdots 2 \mathrm{HF}$ \\
\hline$\nabla^{2} \rho_{\left(H^{3}-{ }_{F}{ }^{4}\right)}$ & $-2,65$ & $-2,51$ & $-2,63$ & $-2,30$ & $-2,66$ & $-2,51$ \\
\hline$\left.\nabla^{2} \rho_{(H}^{5}{ }^{5}{ }^{6}\right)$ & - & $-2,64$ & - & $-2,43$ & - & $-2,66$ \\
\hline$\nabla^{2} \rho_{(\mathrm{C}=\mathrm{C})}$ & $-1,24$ & $-1,23$ & - & - & - & - \\
\hline$\nabla^{2} \rho_{(\mathrm{C}=\mathrm{C})}$ & - & - & $-1,02$ & $-1,00$ & - & - \\
\hline$\nabla^{2} \rho_{(\mathrm{C}-\mathrm{C})}$ & - & - & - & - & $-0,343$ & $-0,318$ \\
\hline$\nabla^{2} \rho_{(\pi \cdots \mathrm{H})}$ & 0,053 & - & 0,050 & 0,019 & - & - \\
\hline$\nabla^{2} \rho_{(\mathrm{nu}-\pi \cdots \mathrm{H})}$ & - & 0,061 & - & - & - & - \\
\hline$\nabla^{2} \rho_{(\mathrm{p}-\pi \cdots \mathrm{H})}$ & - & - & - & - & 0,061 & 0,057 \\
\hline$\nabla^{2} \rho_{\left(\mathrm{F}^{4} \ldots \mathrm{H}^{5}\right)}$ & - & 0,119 & - & 0,137 & - & 0,127 \\
\hline$\nabla^{2} \rho_{\left(F^{6} \cdots H^{2}\right)}$ & - & 0,026 & - & - & - & - \\
\hline$\nabla^{2} \rho_{\left(\mathrm{F}^{3} \cdots \mathrm{H}^{2}\right)}$ & - & - & - & 0,141 & - & - \\
\hline
\end{tabular}

*Valor de $\nabla^{2} \rho$ no ácido fluorídrico livre é - 2,890 e/a ${ }_{0}^{5}$. *Valores de $\nabla^{2} \rho_{(\mathrm{C}=\mathrm{C})}, \nabla^{2} \rho_{(\mathrm{C}=\mathrm{C})}$ e $\nabla^{2} \rho_{(\mathrm{C}-\mathrm{C})}$ nos monômeros $\mathrm{C}_{2} \mathrm{H}_{2}, \mathrm{C}_{2} \mathrm{H}_{4}$ e $\mathrm{C}_{3} \mathrm{H}_{6}$ são $-1,24 ;-1,03$ e $-0,409$ e/ $\mathrm{a}_{0}{ }^{5}$, respectivamente.

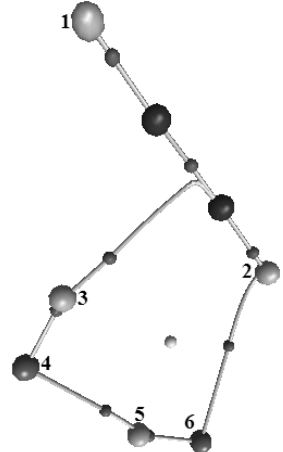

(a)

Figura 3. Pontos críticos de ligação (BCP) para todo o conjunto de interações no complexo de hidrogênio trimolecular $n u-\pi \mathrm{C}_{2} \mathrm{H}_{2} \cdots 2 \mathrm{HF}$ (a)

(H-F) sofre uma variação substancial. Isto pode ser verificado pelos valores de 0,339 e 0,336 e/a ${ }_{0}{ }^{3}$ nos respectivos complexos $\mathrm{C}_{2} \mathrm{H}_{2} \cdots 2 \mathrm{HF}$ e $\mathrm{C}_{2} \mathrm{H}_{4} \cdots 2 \mathrm{HF}$. Com relação às interações intermoleculares, baixos valores de densidade eletrônica foram obtidos, os quais estão na faixa de 0,007-0,04 e/a ${ }_{0}^{3}$. Estes baixos valores de $\rho$ juntamente com os resultados positivos do Laplaciano indicam a formação de ligações de hidrogênio em cada BCP intermolecular. Além das ligações de hidrogênio, os valores do Laplaciano de 0,026 e 0,141 e/a ${ }_{0}^{3}$ listados na Tabela 2 demonstram que as interações terciárias $\left(\mathrm{F}^{6} \cdots \mathrm{H}^{2}\right)$ e $\left(\mathrm{F}^{3} \cdots \mathrm{H}^{2}\right)$ formadas entre a segunda molécula de ácido fluorídrico e os átomos de hidrogênio das moléculas $\mathrm{C}_{2} \mathrm{H}_{4}$ e $\mathrm{C}_{2} \mathrm{H}_{2}$ são caracterizadas como sendo de camada fechada, ou simplesmente ligações de hidrogênio. Estes resultados da análise topológica QTAIM corroboram inteiramente com dados estruturais, devido a uma relação direta entre as quantidades de densidade eletrônica nas ligações de hidrogênio $(\pi \cdots \mathrm{H}),($ nu- $\pi \cdots \mathrm{H}),(\mathrm{p}-\pi \cdots \mathrm{H}),\left(\mathrm{F}^{4} \cdots \mathrm{H}^{5}\right),\left(\mathrm{F}^{6} \cdots \mathrm{H}^{2}\right)$ e $\left(\mathrm{F}^{3} \cdots \mathrm{H}^{2}\right)$ e seus correspondentes valores de distância, conforme indica o coeficiente de correlação $\mathrm{R}^{2}$ de 0,91 , obtido a partir da Equação 5. Ou seja, quanto mais fortemente ligado o sistema intermolecular, maior será a concentração de carga no BCP da sua ligação de hidrogênio.

$$
\mathrm{R}=2,74-29,7 \rho ; \mathrm{R}^{2}=0,91
$$

Como complemento, é plausível considerar que a força da ligação de hidrogênio pode ser reproduzida pela análise de seus modos vibracionais, ${ }^{62}$ os quais também devem ser relacionados com suas densidades eletrônicas. Como já foi mostrado que as distâncias das ligações de hidrogênio se relacionam diretamente com seus respectivos valores da densidade eletrônica, o coeficiente de correlação $\mathrm{R}^{2}$ de 0,94 , obtido pela Equação 6, indica uma relação satisfatória entre os valores de $\rho$ com seus novos modos vibracionais. ${ }^{63}$ Baseado em trabalhos recentes, ${ }^{64}$ certamente os complexos de hidrogênio trimoleculares $\pi$, nu- $\pi$ ou p- $\pi$ representam um conjunto de sistemas mais estáveis quando comparados aos formados bimolecularmente. 
Como a detecção experimental dos modos vibracionais das ligações de hidrogênio na região de infravermelho não é uma tarefa trivial, a análise QTAIM se mostra bastante eficaz na interpretação e caracterização destas frequências.

$$
v^{\mathrm{Str}}=33+5699 \rho ; \mathrm{R}^{2}=0,94
$$

\section{COMPLEXOS DE HIDROGÊNIO HETEROCÍCLICOS}

Conforme reportado na literatura especializada ${ }^{65}$ a formação de interações secundárias tem sido observada em sistemas heterocíclicos intermoleculares. Para os complexos $\mathrm{C}_{2} \mathrm{H}_{4} \mathrm{O} \cdots \mathrm{C}_{2} \mathrm{H}_{2}\left(\right.$ a) e $\mathrm{C}_{2} \mathrm{H}_{4} \mathrm{~S} \cdots \mathrm{C}_{2} \mathrm{H}_{2}$ (b), além das ligações de hidrogênio $(\mathrm{O} \cdots \mathrm{H})$ e $(\mathrm{S} \cdots \mathrm{H})$, os cálculos QTAIM apresentados na Tabela 3 revelam a existência da interação secundária $\left(\mathrm{H}^{2} \cdots \mathrm{C}^{3}\right)$ em (b), cujos respectivos valores de $\rho$ e $\nabla^{2} \rho$ são 0,004 e $0,015 \mathrm{e} / \mathrm{a}_{0}{ }^{5}$. Anterior a esta análise, esta interação secundária era interpretada como sendo formada entre os átomos de hidrogênio $\mathrm{H}^{2}$ do grupo $\mathrm{CH}_{2}$ e a densidade eletrônica da ligação $\pi(\mathrm{C} \equiv \mathrm{C})$ do acetileno. ${ }^{66}$ Todavia, estes novos resultados mostram uma interação direta entre $\mathrm{H}^{2}\left(\mathrm{C}_{2} \mathrm{H}_{4} \mathrm{~S}\right)$ e $\mathrm{C}^{3}\left(\mathrm{C}_{2} \mathrm{H}_{2}\right)$. Desta maneira, o complexo (b) apresenta duas interações intermoleculares, $(\mathrm{S} \cdots \mathrm{H})$ e $\left(\mathrm{H}^{2} \cdots \mathrm{C}^{3}\right)$, enquanto (a) apenas uma, $(\mathrm{O} \cdots \mathrm{H}) .{ }^{67}$ Estruturalmente, foi mostrado que a formação da interação secundária no complexo (b) é mais propícia do que em (a), isso porque as distâncias entre $\mathrm{H}^{2}$ e $\mathrm{C}^{3}$ são compatíveis com a soma dos raios de van der Waals destes átomos. ${ }^{3}$ Isso não significa que o complexo (b) seja mais fortemente ligado e mais estável que (a), mesmo porque as densidades eletrônicas intermoleculares de (b) são muito menores. Além disso, as energias de interação ${ }^{68}$ corrigidas com BSSE (Basis Sets Superposition Error) ${ }^{69}$ e ZPE (Zero Point Energy) ${ }^{70}$ de (a) e (b) são 6,42 e 4,15 kJ mol ${ }^{-1}$, respectivamente.

Analisando as linhas de contorno da densidade eletrônica ilustrada na Figura 4, podem ser observadas a ligação de hidrogênio $(\mathrm{S} \cdots \mathrm{H})$ e a interação secundária $\left(\mathrm{H}^{2} \cdots \mathrm{C}^{3}\right)$ no complexo (b). Pela QTAIM, estas linhas de contorno da densidade eletrônica findam em um ponto crítico molecular, o qual dependendo da estrutura pode ser os núcleos (NA), estruturas anelares ou cíclicas (RCP), compartimento ou uma região de interseção entre átomos $(\mathrm{CCP})^{71}$, ou ligações químicas (BCP).$^{72}$

Além da densidade eletrônica e do Laplaciano, também foram calculadas as elipicidades $\varepsilon$ para estas linhas de contorno, cujos valores são apresentados nas Tabelas 3 e 4 . Na molécula de acetileno, a densidade eletrônica de 0,401 e/a ${ }_{0}^{3}$ indica uma alta concentração de carga em sua ligação $\pi(\mathrm{C} \equiv \mathrm{C})$. Porém, é bem conhecido que o caráter simétrico do acetileno provoca um valor praticamente nulo $(\varepsilon=6,6$ $\left.10^{-15}\right)$ de elipicidade em $(\mathrm{C} \equiv \mathrm{C})$, sendo este um dos casos excepcionais em que $\rho$ e $\varepsilon$ se mostram totalmente descorrelacionados. ${ }^{73}$ Entretanto, observa-se um pequeno aumento de $\varepsilon$ na ligação $\pi(\mathrm{C} \equiv \mathrm{C})$ dos complexos (a) e (b), cujos valores são 0,002 e 0,003, respectivamente. Como é bem estabelecido que os valores das elipicidades de 0,00; 0,23 e 0,45 indicam a concentração de carga nas ligações carbono-carbono das moléculas de etano, benzeno e eteno, respectivamente, ${ }^{11}$ pode-se assumir que a mudança na linearidade do eixo internuclear $\mathrm{H}-\mathrm{C}-\mathrm{C}-\mathrm{H}$ do acetileno seja responsável por esse aumento de $\varepsilon$.

Para sistemas heterocíclicos trimoleculares, ${ }^{74}$ os parâmetros topológicos da QTAIM têm sido decisivos na determinação da configuração mais estável. Na Figura 5, são apresentadas três potenciais geometrias para o complexo de hidrogênio trimolecular $\mathrm{C}_{2} \mathrm{H}_{4} \mathrm{O} \cdots 2 \mathrm{HF}$, onde a presença de duas moléculas de ácido fluorídrico tem por objetivo romper a estrutura heterocíclica da oxirana com mais eficiência em relação ao complexo bimolecular $\mathrm{C}_{2} \mathrm{H}_{4} \mathrm{O} \cdots \mathrm{HF}$. Na Tabela 5 são apresentados os valores das densidades eletrônicas e dos Laplacianos para as ligações de hidrogênio $(\mathrm{O} \cdots \mathrm{H})$ e $\left(\mathrm{F}^{4} \cdots \mathrm{F}^{5}\right)$, como também de uma possível interação terciária $\left(\mathrm{F}^{6} \cdots \mathrm{H}^{1}\right)$. Em comparação ao monô-
Tabela 3. Valores das densidades eletrônicas $\left(\rho\right.$ em e/a $\left.{ }_{0}{ }^{3}\right)$, Laplacianos $\left(\nabla^{2} \rho\right.$ em e/ $\mathrm{a}_{0}{ }^{5}$ ) e elipicidades $(\varepsilon)$ nos $\mathrm{BCP}$ das principais ligações químicas dos

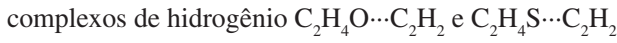

\begin{tabular}{lcccc}
\hline \multirow{2}{*}{ Complexos } & \multirow{3}{*}{$\mathrm{BCP}$} & \multicolumn{3}{c}{ Parâmetros topológicos QTAIM } \\
& & $\rho$ & $\nabla^{2} \rho$ & $\varepsilon=\lambda_{1} / \lambda_{2}-1$ \\
\hline $\mathrm{C}_{2} \mathrm{H}_{4} \mathrm{O} \cdots \mathrm{C}_{2} \mathrm{H}_{2}$ & $(\mathrm{H}-\mathrm{C})$ & 0,287 & $-1,159$ & 0,000 \\
& $(\mathrm{C} \equiv \mathrm{C})$ & 0,402 & $-1,168$ & 0,002 \\
& $(\mathrm{C}-\mathrm{C})$ & 0,259 & $-0,565$ & 0,241 \\
& $(\mathrm{C}-\mathrm{Y})$ & 0,248 & $-0,380$ & 0,670 \\
& $\left(\mathrm{C}-\mathrm{H}^{2}\right)$ & 0,288 & $-1,056$ & 0,036 \\
& $\left(\mathrm{C}-\mathrm{H}^{1}\right)$ & 0,287 & $-1,052$ & 0,037 \\
& $(\mathrm{O} \cdots \mathrm{H})$ & 0,019 & 0,052 & 0,015 \\
& $\left(\mathrm{H}^{2} \cdots \mathrm{C}^{3}\right)$ & - & - & - \\
$\mathrm{C}_{2} \mathrm{H}_{4} \mathrm{~S} \cdots \mathrm{C}_{2} \mathrm{H}_{2}$ & $\left(\mathrm{H}^{2} \cdots \mathrm{C}^{4}\right)$ & - & - & - \\
& $(\mathrm{H}-\mathrm{C})$ & 0,288 & $-1,152$ & 0,000 \\
& $(\mathrm{C} \equiv \mathrm{C})$ & 0,402 & $-1,165$ & 0,003 \\
& $(\mathrm{C}-\mathrm{C})$ & 0,264 & $-0,608$ & 0,168 \\
& $(\mathrm{C}-\mathrm{Y})$ & 0,157 & $-0,143$ & 0,416 \\
& $\left(\mathrm{C}-\mathrm{H}^{2}\right)$ & 0,287 & $-1,044$ & 0,019 \\
& $\left(\mathrm{C}-\mathrm{H}^{1}\right)$ & 0,286 & $-1,037$ & 0,018 \\
& $(\mathrm{~S} \cdots \mathrm{H})$ & 0,009 & 0,027 & 0,028 \\
& $\left(\mathrm{H}^{2} \cdots \mathrm{C}^{3}\right)$ & 0,004 & 0,015 & 0,832 \\
& $\left(\mathrm{H}^{2} \cdots \mathrm{C}^{4}\right)$ & - & - & - \\
\hline
\end{tabular}

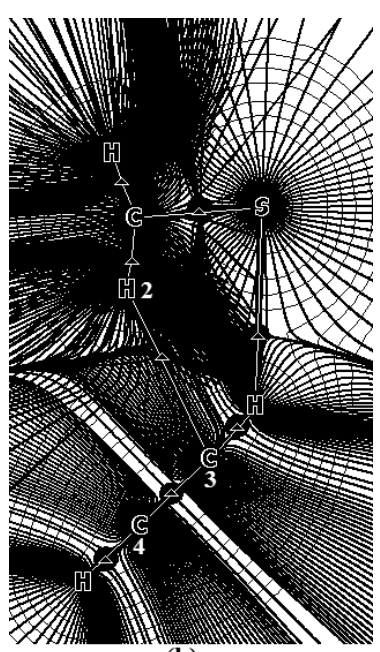

(b)

Figura 4. Mapa da superfície de densidade eletrônica indicando os caminhos de interação internuclear e os pontos críticos de ligação $(B C P)$ no complexo de hidrogênio $\mathrm{C}_{2} \mathrm{H}_{4} \mathrm{~S} \cdots \mathrm{C}_{2} \mathrm{H}_{2}(\boldsymbol{b})$

Tabela 4. Valores das densidades eletrônicas $\left(\rho\right.$ em e $\left./ \mathrm{a}_{0}{ }^{3}\right)$, Laplacianos $\left(\nabla^{2} \rho\right.$ em e/a ${ }_{0}^{5}$ ) e elipicidades $(\varepsilon)$ nos $\mathrm{BCP}$ das principais ligações químicas dos monômeros $\mathrm{C}_{2} \mathrm{H}_{2}, \mathrm{C}_{2} \mathrm{H}_{4} \mathrm{O}$ e $\mathrm{C}_{2} \mathrm{H}_{4} \mathrm{~S}$

\begin{tabular}{lllll}
\hline \multirow{2}{*}{ Monômeros } & \multirow{2}{*}{ BCP } & \multicolumn{3}{l}{ Parâmetros topológicos QTAIM } \\
& & $\rho$ & $\nabla^{2} \rho$ & $\varepsilon=\lambda_{\perp} / \lambda_{2}-1$ \\
\hline $\mathrm{C}_{2} \mathrm{H}_{2}$ & $(\mathrm{H}-\mathrm{C})$ & 0,290 & $-1,140$ & 0,000 \\
& $(\mathrm{C} \equiv \mathrm{C})$ & 0,401 & $-1,116$ & 0,000 \\
$\mathrm{C}_{2} \mathrm{H}_{4} \mathrm{O}$ & $(\mathrm{C}-\mathrm{Y})$ & 0,250 & $-0,372$ & 0,595 \\
& $\left(\mathrm{C}-\mathrm{H}^{2}\right)$ & 0,255 & $-0,976$ & 0,033 \\
& $\left(\mathrm{C}-\mathrm{H}^{1}\right)$ & 0,255 & $-0,976$ & 0,033 \\
$\mathrm{C}_{2} \mathrm{H}_{4} \mathrm{~S}$ & $(\mathrm{C}-\mathrm{Y})$ & 0,158 & $-0,148$ & 0,390 \\
& $\left(\mathrm{C}-\mathrm{H}^{2}\right)$ & 0,285 & $-1,034$ & 0,020 \\
& $\left(\mathrm{C}-\mathrm{H}^{1}\right)$ & 0,285 & $-1,034$ & 0,020 \\
\hline
\end{tabular}


mero do ácido fluorídrico, sua densidade eletrônica nos isômeros (a), (b) e (c) diminui em -0,036; -0,032 e -0,040 e/a ${ }_{0}^{3}$ nas ligações $\left(\mathrm{H}^{5}-\mathrm{F}^{6}\right)$, como também $-0,071$ e $-0,078$ e/a ${ }_{0}^{3} \mathrm{em}\left(\mathrm{H}^{3}-\mathrm{F}^{4}\right)$, respectivamente. Isso nos leva a considerar que $\left(\mathrm{H}^{3}-\mathrm{F}^{4}\right)$ apresenta um enfraquecimento, ou em termos dos conceitos QTAIM, simplesmente uma tendência de formar uma interação de camada fechada, em especial no isômero (c). Além disso, como a densidade eletrônica diminui no BCP da ligação $\left(\mathrm{H}^{3}-\mathrm{F}^{4}\right)$, automaticamente seu Laplaciano de $-1,844$ e/a ${ }_{0}^{5}$ torna-se menos negativo se comparado com o valor de $-2,754 \mathrm{e} / \mathrm{a}_{0}{ }^{5}$ para o ácido fluorídrico monomérico.

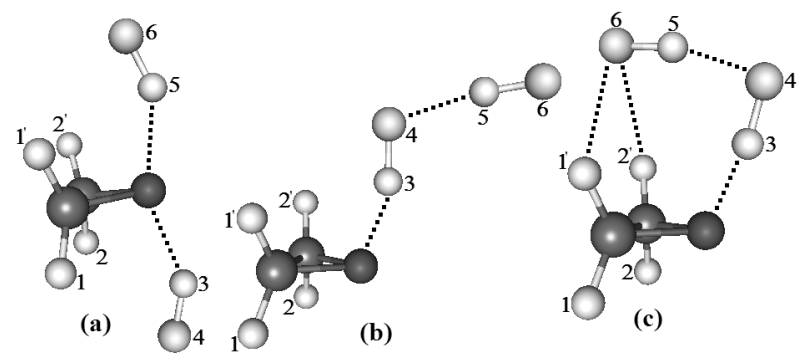

Figura 5. Geometrias otimizadas dos isômeros (a), (b) e (c) para o complexo de hidrogênio $\mathrm{C}_{2} \mathrm{H}_{4} \mathrm{O} \cdots 2 \mathrm{HF}$ usando o nível de teoria B3LYP/aug-cc-pVDZ

Tabela 5. Valores das densidades eletrônicas $\left(\rho\right.$ em e $\left./ \mathrm{a}_{0}{ }^{3}\right)$ e Laplacianos $\left(\nabla^{2} \rho\right.$ em e/ $\mathrm{a}_{0}{ }^{5}$ ) nos BCP para as ligações de hidrogênio nas três geometrias do complexo de hidrogênio $\mathrm{C}_{2} \mathrm{H}_{4} \mathrm{O} \cdots 2 \mathrm{HF}$

Geometrias do complexo $\mathrm{C}_{2} \mathrm{H}_{4} \mathrm{O} \cdots 2 \mathrm{HF}$ e parâmetros topológicos

\begin{tabular}{lcccccc} 
Ligações & \multicolumn{2}{c}{ (a) } & \multicolumn{2}{c}{ (b) } & \multicolumn{2}{c}{ (c) } \\
& $\rho$ & $\nabla^{2} \rho$ & $\mathrm{r}$ & $\nabla^{2} \rho$ & $\mathrm{r}$ & $\nabla^{2} \rho$ \\
\hline$(\mathrm{O} \cdots \mathrm{H})$ & 0,042 & 0,153 & 0,064 & 0,176 & 0,068 & 0,171 \\
$\left(\mathrm{~F}^{4} \cdots \mathrm{F}^{5}\right)$ & - & - & 0,036 & 0,155 & 0,042 & 0,168 \\
$\left(\mathrm{~F}^{6} \cdots \mathrm{H}^{1}\right)$ & - & - & - & - & 0,005 & 0,022 \\
$\left(\mathrm{H}^{3}-\mathrm{F}^{4}\right)$ & - & - & 0,294 & $-1,984$ & 0,287 & $-1,844$ \\
& 0,329 & & $(-0,071)$ & & $(-0,078)$ & \\
$\left(\mathrm{H}^{5}-\mathrm{F}^{6}\right)$ & $(-0,036)$ & $-2,554$ & 0,333 & $-2,767$ & 0,325 & $-2,623$ \\
\hline
\end{tabular}

*Valores de $\rho$ e $\nabla^{2} \rho$ para o HF monomérico são 0,367 e/a ${ }_{0}^{3}$ e $-2,754$ e/a ${ }_{0}{ }^{5}$, respectivamente. *Em parênteses são apresentadas as variações na densidade eletrônica da ligação H-F quando ocorre a formação dos complexos de hidrogênio (a), (b) e (c).

A respeito da formação das ligações de hidrogênio $(\mathrm{O} \cdots \mathrm{H})$, estas possuem baixas concentrações de densidade eletrônica, cujos valores estão na faixa de 0,042-0,068 e/a ${ }_{0}{ }^{3}$. Ademais, as densidades eletrônicas nas ligações de hidrogênio $\left(\mathrm{F}^{4} \cdots \mathrm{F}^{5}\right)$ apresentam valores ainda mais baixos, os quais são 0,036 e 0,042 e/a ${ }_{0}^{3}$ para os isômeros (b) e (c), respectivamente. Todavia, pode ser observado nos BCP e caminhos de ligação ilustrados na Figura 6 que os cálculos QTAIM confirmam a existência da interação terciária entre o flúor $\left(\mathrm{F}^{6}\right)$ e os átomos de hidrogênio axiais $\left(\mathrm{H}^{1} \mathrm{e} \mathrm{H}^{2}\right)$ no complexo $(\mathbf{c})$, cujos valores de $\rho$ e $\nabla^{2} \rho$ são 0,005 e 0,022 e/a ${ }_{0}^{5}$, respectivamente. Do ponto de vista energético, cálculos B3LYP/aug-ccpVDZ mostram que as respectivas energias de interação corrigidas com valores de $\mathrm{BSSE}^{68} \mathrm{e} \mathrm{ZPE}^{69}$ para os complexos (a), (b) e (c) são 49,25; 5,42 e 60,82 $\mathrm{kJ} \mathrm{mol}^{-1}$. Desta maneira, podemos afirmar que a maior energia de estabilização no isômero $(\mathbf{c})$ se deve à formação das ligações de hidrogênio $(\mathrm{O} \cdots \mathrm{H})$ e $\left(\mathrm{F}^{4} \cdots \mathrm{F}^{5}\right)$, mas também da interação secundária $\left(\mathrm{F}^{6} \cdots \mathrm{H}^{1}\right)$.

\section{Complexos de di-hidrogênio}

É bem estabelecido que as ligações de hidrogênio são consideradas uma das interações não covalentes mais importantes da natureza. ${ }^{75}$

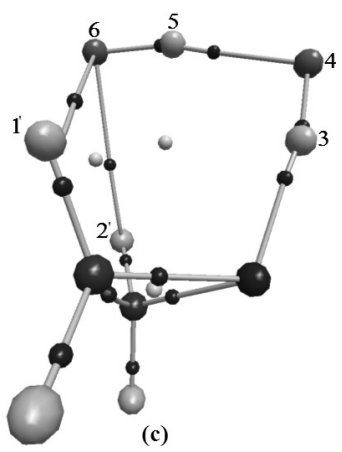

Figura 6. Pontos críticos de ligação (BCP) e caminhos de densidade eletrônica para o isômero (c) do complexo de hidrogênio $\mathrm{C}_{2} \mathrm{H}_{4} \mathrm{O} \cdots 2 \mathrm{HF}$

A interação entre um doador de próton $\left(\mathrm{H}^{+\delta}\right)$ e um centro contendo alta densidade eletrônica (pares de elétrons desemparelhados ou ligações $\pi$ ) compõe a definição mais moderna a respeito da ligação de hidrogênio. ${ }^{76}$ Todavia, há alguns anos Crabtree e seus colaboradores descobriram um sistema intermolecular formado por uma interação muito distinta da tradicional ligação de hidrogênio. ${ }^{77}$ Este novo sistema intermolecular é conhecido como complexo de di-hidrogênio, e sua principal característica está na capacidade do átomo de hidrogênio agir como aceitador de prótons. ${ }^{78,79}$ Por definição, complexos de dihidrogênio caracterizam-se por interações não covalentes formadas por doadores de hidrogênio $\mathrm{H}^{+\delta}$ (átomos de hidrogênio presentes nas ligações $\mathrm{N}-\mathrm{H}, \mathrm{O}-\mathrm{H}, \mathrm{C}-\mathrm{H}, \mathrm{H}-\mathrm{F}$ e $\mathrm{H}-\mathrm{Cl}$ ) e grupos hidretos $\mathrm{H}^{-\delta}$ provenientes de metais alcalinos ou alcalinos terrosos ${ }^{80-82} \mathrm{Em}$ alguns exemplos, não apenas cálculos $a b$ initio ou funcionais híbridos têm apresentado bons resultados no estudo de ligações de di-hidrogênio, mas a QTAIM se destaca como ferramenta obrigatória devido a sua capacidade de localizar BCP na região internuclear entre $\mathrm{H}^{+\delta} \mathrm{e} \mathrm{H}^{-\delta} .83,84$

Pela própria definição das ligações de di-hidrogênio, alguns trabalhos mostram que o hidreto de berílio $\left(\mathrm{BeH}_{2}\right)$ pode interagir com espécies ácidas ${ }^{85}$ como, por exemplo, o ácido fluorídrico. Sabe-se que a ligação $(\mathrm{Be}-\mathrm{H})$ é levemente alterada devido à formação do complexo, embora também se observe que a distância da ligação (H-F) é drasticamente alongada em ligações de di-hidrogênio $\left(\mathrm{H}^{+\delta} \cdots \mathrm{H}^{-\delta}\right)$ mais fortes. Semelhantemente aos sistemas trimoleculares $\pi$, nu- $\pi$ ou p- $\pi$, os complexos de di-hidrogênio também podem ser estudados através do deslocamento vibracional ocorrido nas frequências de estiramento, ou mais comumente conhecido como efeito batocrômico. Pela aplicação do nível de teoria B3LYP/6-311++G(3d,3p), na Tabela 6 são apresentados os valores das frequências de estiramento e intensidades de absorção das ligações de di-hidrogênio, como também os valores dos efeitos batocrômicos $\left(\Delta v_{\mathrm{HX}}^{\mathrm{Str}}\right)$ calculados para as ligações $\mathrm{HX}(\mathrm{X}$ $=\mathrm{F}, \mathrm{Cl}, \mathrm{CN}$, e CCH) dos complexos $\mathrm{BeH}_{2} \cdots \mathrm{HF}, \mathrm{BeH}_{2} \cdots \mathrm{HCl}, \mathrm{BeH}_{2} \cdots$ $\mathrm{HCN}, \mathrm{BeH}_{2} \cdots \mathrm{HNC}$ e $\mathrm{BeH}_{2} \cdots \mathrm{HCCH}^{86}{ }^{8}$ Como o efeito batocrômico é provocado pela transferência de carga proveniente da interação entre os orbitais HOMO (Highest Occupied Molecular Orbital) e LUMO (Lowest Unoccupied Molecular Orbital) das espécies doadoras e receptoras de prótons, respectivamente, a relação entres os valores de $\Delta v_{\mathrm{HX}}^{\text {Str }}$ e de transferência de carga QTAIM $\left(\Delta \mathrm{Q}_{\mathrm{HX}}^{\mathrm{QTAIM}}\right)$ é descrita conforme a Equação 7, pela qual foi obtido um coeficiente de correlação $\mathrm{R}^{2}$ de 0,99 .

$$
\Delta \mathrm{Q}_{\mathrm{HX}}^{\mathrm{QTAIM}}=1,36 \Delta v_{\mathrm{HX}}^{\mathrm{Str}}-0,008 ; \mathrm{R}^{2}=0,99
$$

Calculada a partir das cargas atômicas QTAIM (Equação 8), ${ }^{87}$ a transferência de carga $\Delta \mathrm{Q}_{\mathrm{HX}}^{\mathrm{QTAM}}$ é considerada um parâmetro muito eficiente para justificar os efeitos batocrômicos nos complexos de di-hidrogênio $\mathrm{BeH}_{2} \cdots \mathrm{HX}$. 
Tabela 6. Frequências de estiramento (v), intensidades de absorção (I) e deslocamentos batocrômicos $\left(\Delta v_{\mathrm{HX}}^{\mathrm{Str}}\right)$ nas ligações $\mathrm{HX}(\mathrm{X}=\mathrm{F}, \mathrm{Cl}, \mathrm{CN}$, e $\mathrm{CCH})$ dos sistemas $\mathrm{BeH}_{2} \cdots \mathrm{HX}$. Todos os valores foram obtidos através do cálculo B3LYP/6-311++G(3d,3p)

\begin{tabular}{lcccc}
\hline \multirow{2}{*}{ Parâmetros } & \multicolumn{5}{c}{ Complexos de di-hidrogênio $\mathrm{BeH}_{2} \cdots \mathrm{HX}$} \\
& $\mathrm{X}=\mathrm{F}$ & $\mathrm{X}=\mathrm{Cl}$ & $\mathrm{X}=\mathrm{CN}$ & $\mathrm{X}=\mathrm{CCH}$ \\
\hline $\mathrm{v}_{\left(\mathrm{H}^{-\delta} \cdots \mathrm{H}^{+\delta}\right)}^{\mathrm{Str}}$ & 173,4 & 115,5 & 97,7 & 69,5 \\
$\mathrm{I}_{\left(\mathrm{H}^{-\delta} \cdots \mathrm{H}^{+\delta}\right)}^{\mathrm{Str}}$ & 1,9 & 3,0 & 0,7 & 0,3 \\
$\Delta \mathrm{v}_{\mathrm{HX}}^{\mathrm{Str}}$ & $-240,0$ & $-148,7$ & $-62,5$ & $-15,3$ \\
$\mathrm{I}_{\mathrm{HX}, \mathrm{c} /}^{\mathrm{Str}} \mathrm{I}_{\mathrm{HX}, \mathrm{m}}^{\mathrm{Str}}$ & 6,2 & 8,9 & 3,2 & 2,6 \\
\hline
\end{tabular}

*Valores de $v$ e I são dados em $\mathrm{cm}^{-1} \mathrm{e} \mathrm{km} \mathrm{mol}^{-1}$, respectivamente.

$$
\mathrm{Q}^{\mathrm{QTAIM}}={\mathrm{Z}-{ }_{\Omega}}_{\rho} \rho \mathrm{d} \tau
$$

Entretanto, sabe-se que os efeitos batocrômicos não são únicos, e que os deslocamentos vibracionais para a região do azul ou efeitos hipsocrômicos também são observados em alguns sistemas intermoleculares. ${ }^{88}$ Ao contrário do efeito batocrômico em que se observa uma decaída na frequência de estiramento na ligação doadora de próton, no efeito hipsocrômico a frequência do oscilador $(\mathrm{H}-\mathrm{X})$ é deslocada para valores mais elevados no espectro de infravermelho. ${ }^{89,90}$ Foi neste sentido que Hobza ${ }^{91}$ e coautores propuseram os primeiros estudos teóricos sobre deslocamentos vibracionais hipsocrômicos em complexos de hidrogênio. ${ }^{92}$

Considerando que os hidretos $\mathrm{BeH}_{2}, \mathrm{LiH}, \mathrm{MgH}_{2}$ e NaH são capazes de formar ligações de di-hidrogênio estáveis, como também que a frequência de estiramento da ligação $(\mathrm{C}-\mathrm{H})$ do fluorofórmio $\left(\mathrm{HCF}_{3}\right)$ se desloca para valores mais elevados no espectro devido à formação da ligação de hidrogênio, recentemente foi mostrado que estas moléculas podem formar ligações de di-hidrogênio $\left(\mathrm{H}^{+\delta} \ldots \mathrm{H}^{-\delta}\right)$ com deslocamentos hipsocrômicos nos complexos $\mathrm{BeH}_{2} \cdots \mathrm{HCF}_{3}(\mathbf{a})$, $\mathrm{LiH} \cdots \mathrm{HCF}_{3}(\mathbf{b}), \mathrm{MgH}_{2} \cdots \mathrm{HCF}_{3}$ (c) e $\mathrm{NaH} \cdots \mathrm{HCF}_{3}(\mathbf{d}) .{ }^{93}$ Para estes sistemas, a QTAIM foi aplicada com o propósito de obter parâmetros topológicos $\left(\rho\right.$ e $\left.\nabla^{2} \rho\right)$ que justificassem a formação destes efeitos hipsocrômicos. Como esperado, os baixos valores de densidades eletrônicas como também os resultados positivos dos Laplacianos apresentados na Tabela 7 indicam que as ligações de di-hidrogênio $\left(\mathrm{H}^{+\delta} \ldots \mathrm{H}^{-\delta}\right)$ são identificadas como interações de camada fechada. Para o complexo (a), a Figura 7 ilustra o conjunto de BCP, as linhas de contorno que formam a isossuperfície de densidade eletrônica e o caminho molecular que caracteriza a formação da ligação de di-hidrogênio entre os átomos $\mathrm{H}^{-\delta}\left(\mathrm{BeH}_{2}\right) \mathrm{e} \mathrm{H}^{+\delta}\left(\mathrm{HCF}_{3}\right)$. Como é conhecido que a densidade de carga calculada no BCP intermolecular pode ser usada para estimar a força das ligações de di-hidrogênio $\left(\mathrm{H}^{-\delta} \cdots \mathrm{H}^{+\delta}\right),{ }^{94}$ (a) é o complexo de di-hidrogênio mais fracamente ligado, enquanto (b), (c) e (d) são mais fortemente estabilizados.

Em se tratando de interações com densidade de carga compartilhada, percebe-se que a densidade eletrônica na ligação $\left(\mathrm{C}-\mathrm{H}^{+\delta}\right)$ do fluorofórmio é mais elevada em comparação às calculadas nas ligações de di-hidrogênio $\left(\mathrm{H}^{-}{ }^{-} \cdots \mathrm{H}^{+\delta}\right)$. O acúmulo de densidade eletrônica (Figura 8) na ligação $\left(\mathrm{C}-\mathrm{H}^{+\delta}\right)$ é justificado por $\nabla^{2} \rho<$ 0 , embora os valores de $-1,261 ;-1,265$ e $-1,273 \mathrm{a}_{0}{ }^{5}$ sejam menos negativos nos complexos (a), (b), (c) e (d) se comparados com o fluorofórmio monomérico, no qual o valor de $\nabla^{2} \rho$ é $-2,124 \mathrm{a}_{0}{ }^{5}$. De fato, não há como contestar que a densidade eletrônica pode ser usada para interpretar a estabilização intermolecular, mas as
Tabela 7. Valores das densidades eletrônicas $\left(\rho\right.$ em a $\left._{0}{ }^{3}\right)$ e Laplacianos $\left(\nabla^{2} \rho\right.$ em $\left.\mathrm{a}_{0}^{5}\right)$ nos BCP dos complexos de di-hidrogênio $\mathrm{BeH}_{2} \cdots \mathrm{HCF}_{3}(\mathbf{a})$, $\mathrm{MgH}_{2} \cdots \mathrm{HCF}_{3}(\mathbf{b}), \mathrm{LiH} \cdots \mathrm{HCF}_{3}(\mathbf{c})$ and $\mathrm{NaH} \cdots \mathrm{HCF}_{3}(\mathbf{d})$

\begin{tabular}{lcccc}
\hline \multirow{2}{*}{ Parâmetros } & \multicolumn{5}{c}{ Complexos de di-hidrogênio } \\
& $($ a) & $($ b $)$ & $($ c $)$ & $($ d $)$ \\
\hline$\rho\left(\mathrm{H}_{\mathrm{b}}^{-\delta} \ldots \mathrm{H}^{+\delta}\right)$ & 0,007 & 0,010 & 0,015 & 0,016 \\
$\nabla^{2} \rho\left(\mathrm{H}_{\mathrm{b}}^{-\delta} \ldots \mathrm{H}^{+\delta}\right)$ & 0,018 & 0,023 & 0,032 & 0,033 \\
$\rho\left(\mathrm{C}-\mathrm{H}^{+\delta}\right)$ & $0,313(0,302)$ & $0,312(0,302)$ & $0,311(0,302)$ & $0,310(0,302)$ \\
$\Delta \rho\left(\mathrm{C}-\mathrm{H}^{+\delta}\right)$ & 0,011 & 0,010 & 0,009 & 0,008 \\
$\nabla^{2} \rho\left(\mathrm{C}-\mathrm{H}^{+\delta}\right)$ & $-1,261$ & $-1,265$ & $-1,2730$ & $-1,2660$ \\
& $(-2,124)$ & $(-2,124)$ & $(-2,124)$ & $(-2,124)$ \\
$\Delta \mathrm{Q}_{\mathrm{HCF} 3}^{\text {QTAIM }}$ & $-0,014$ & $-0,025$ & $-0,046$ & $-0,056$ \\
\hline
\end{tabular}

*Valores de $\Delta Q_{\mathrm{HCF}}^{\mathrm{QTAIM}}$ são dados em unidades eletrônicas (e.u.). *Os valores de densidade eletrônica e Laplaciano da ligação $\mathrm{C}-\mathrm{H}^{+\delta}$ do fluorofórmio livre são dados entre parênteses.

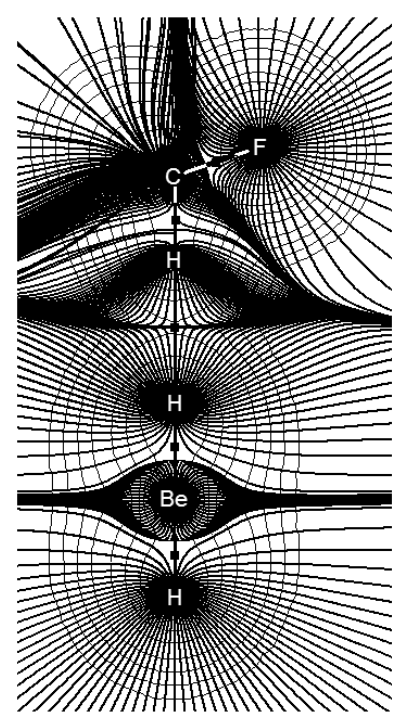

(a)

Figura 7. Mapas de linhas de contorno de densidade eletrônica (linhas finas) e BCP (pequenos quadrados) do complexo de di-hidrogênio $\mathrm{BeH}_{2} \cdots \mathrm{HCF}_{3}(\boldsymbol{a})$

cargas atômicas calculadas pelo protocolo QTAIM (Equação 8) e, consequentemente, as transferências de carga podem ser usadas para investigar a força das ligações de di-hidrogênio $\left(\mathrm{H}^{-} \cdots \mathrm{H}^{+\delta}\right)$. Neste sentido, a análise é concentrada na avaliação da transferência de carga total $\triangle \mathrm{Q}_{\mathrm{HCF} 3}^{\mathrm{QTAIM}}$ na molécula de $\mathrm{HCF}_{3}$, cujos valores também estão listados na Tabela 7. O coeficiente de correlação $\mathrm{R}^{2}$ de 0,99 , descrito pela Equação 9, mostra uma ótima relação entre a transferência de carga $\Delta \mathrm{Q}_{\mathrm{HCF} 3}^{\mathrm{QTAIM}}$ e os efeitos hipsocrômicos nas ligações $\left(\mathrm{C}-\mathrm{H}^{+\delta}\right)$ do fluorofórmio. Deste modo, demonstra-se que os efeitos batocrômicos em (a) e hipsocrômicos em (b), (c) e (d) podem ser explicados pelas diminutas e elevadas transferências de carga, cujos valores são $-0,014 ;-0,025 ;-0,046$ e -0,056 e.u., respectivamente.

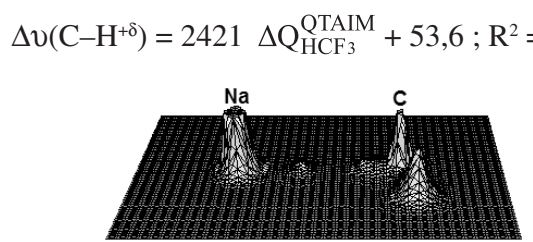

(d)

Figura 8. Mapa do relevo da densidade eletrônica do complexo de dihidrogênio $\mathrm{NaH} \cdots \mathrm{HCF}_{3}(\boldsymbol{d})$ 


\section{CONCLUSÕES}

Conforme proposta de R. Bader, o formalismo da QTAIM foi desenvolvido para ser utilizado no estudo da ligação química e na interpretação de fenômenos moleculares. Entretanto, os fundamentos da QTAIM também têm sido aplicados na investigação de complexos de hidrogênio e complexos de di-hidrogênio. A caracterização destas interações através da localização de BCP proporciona ao protocolo QTAIM quantificar a densidade eletrônica intermolecular, pela qual as ligações de hidrogênio ${ }^{95}$ e ligações de di-hidrogênio ${ }^{96}$ são consideradas interações de camada fechada, devido à carga ser concentrada em núcleos separados, tais como ligações $\pi$ ou pares de elétrons desemparelhados e ácidos monopróticos. Este argumento tem se mostrado decisivo na caracterização de ligações de hidrogênio tradicionais $\pi$, não usuais e pseudo- $\pi$, como no caso dos complexos $\mathrm{C}_{2} \mathrm{H}_{4} \cdots 2 \mathrm{HF}, \mathrm{C}_{2} \mathrm{H}_{2} \cdots 2 \mathrm{HF}$ e $\mathrm{C}_{3} \mathrm{H}_{6} \cdots 2 \mathrm{HF}$. Os cálculos topológicos também foram importantes para caracterizar interações secundárias em complexos de hidrogênio heterocíclicos, como no caso do sistema $\mathrm{C}_{2} \mathrm{H}_{4} \mathrm{~S} \cdots \mathrm{C}_{2} \mathrm{H}_{2}$, em que ao invés da ligação $\pi$ ser a fonte de densidade eletrônica da interação secundária, esta é formada entre os átomos de hidrogênio axiais do anel $\mathrm{C}_{2} \mathrm{H}_{4} \mathrm{~S}$ e o carbono acetilênico. Embora seja bem conhecido que as ligações de di-hidrogênio sejam observadas experimentalmente, os cálculos QTAIM se mostram eficientes não apenas para caracterizar a ligação $\left(\mathrm{H}^{+\delta} \ldots \mathrm{H}^{-\delta}\right)$, mas também para interpretar fenômenos observáveis como, por exemplo, os deslocamentos batocrômicos e hipsocrômicos na região do infravermelho. Estes dois efeitos vibracionais são característicos de sistemas formados por ligações de hidrogênio, mas também têm sido verificados nas ligações de di-hidrogênio ${ }^{97}$ dos complexos $\mathrm{BeH}_{2} \cdots \mathrm{HCF}_{3}, \mathrm{MgH}_{2} \cdots \mathrm{HCF}_{3}, \mathrm{LiH} \cdots$ $\mathrm{HCF}_{3}$ e NaH $\cdots \mathrm{HCF}_{3}$. A investigação dos deslocamentos batocrômicos e hipsocrômicos é desenvolvida pela QTAIM através da quantificação das densidades eletrônicas nas ligações das espécies doadoras de prótons, as quais são reconhecidas como sendo interações compartilhadas.

Além disso, a interação entre os orbitais HOMO e LUMO dos respectivos aceitadores e doadores de prótons pode ser avaliada pelas cargas atômicas QTAIM, que são calculadas pela relação entre a integração tridimensional da densidade eletrônica e o número atômico de cada elemento do sistema molecular. Em termos de algoritmos desenvolvidos para calcular partições de cargas atômicas, sabe-se que estas não têm fundamento físico, pois não são justificadas pela equação de Schrödinger. ${ }^{98}$ Todavia, as cargas atômicas QTAIM se diferenciam de qualquer outro método, pois embora não haja uma expressão analítica que descreva explicitamente a densidade eletrônica, esta observável possui uma interpretação física clara, ${ }^{99}$ e pode ser observada experimentalmente como, por exemplo, nos ensaios cristalográficos de raios-X. Apesar de ser uma metodologia teórica bem estabelecida, ${ }^{100,101}$ a QTAIM ainda é muito debatida, ${ }^{102}$ mas se encontra em pleno desenvolvimento. Uma das novas vertentes é o formalismo QTPOS (Quantum Theory of Proper Open Systems), ${ }^{103}$ desenvolvido para estudar sistemas de camadas abertas com uma interpretação mais íntima da superfície de fluxo zero.

\section{AGRADECIMENTOS}

Ao apoio das agências de fomento CAPES, CNPq e FACEPE.

\section{REFERÊNCIAS}

1. Pauling, L.; Proc. Natl. Acad. Sci. U.S.A. 1928, 14, 359; Pauling, L.; J. Am. Chem. Soc. 1935, 57, 2680; Pauling, L.; Niemann, C.; J. Am. Chem. Soc. 1939, 61, 1860.

2. Pauling, L.; Nature of the Chemical Bond and the Structure of Molecules and Crystals: An Introduction to Modern Structural Chemistry, Cornell University Press: Nova York, 1939.

3. Bondi, A.; J. Phys. Chem. 1964, 68, 441.
4. Rowland, R. S.; Taylor, R.; J. Phys. Chem. 1996, 100, 7384.

5. Mandal, P. K.; Arunan, E.; J. Chem. Phys. 2001, 114, 3880.

6. Karan, N. K.; Arunan, E.; J. Mol. Struct. 2004, 688, 203.

7. Kojić-Prodić, B.; Molčanov, K.; Acta Chim. Slov. 2008, 55, 692.

8. Frisch, M. J.; Pople, J. A.; Binkley, J. S.; J. Chem. Phys. 1984, 80, 3265.

9. Feller, D.; Davidson, E. R.; Rev. Comput. Chem. 2007, 1, 1.

10. Vasconcellos, M. L. A. A.; Lima Jr., C. G.; Quim. Nova 2009, 32, 244.

11. Bader, R. F. W.; Atoms in Molecules. A Quantum Theory, Clarendon Press: Oxford, 1990.

12. Bader, R. F. W.; Chem. Rev. 1991, 91, 893.

13. Cortés-Guzmán, F.; Bader, R. F. W.; Coord. Chem. Rev. 2005, 249, 633.

14. Grabowski, S.J .; Sokalski, W. A.; Leszczynski, J.; J. Phys. Chem. A 2004, 108, 1806.

15. Oliveira, B. G.; Araújo, R. C. M. U.; Carvalho, A. B.; Ramos, M. N.; Struct. Chem. 2009, 20, 663.

16. Teschl, G.; Mathematical Methods in Quantum Mechanics with Applications to Schrödinger Operators, American Mathematical Society: Providence, 2009.

17. Bader, R. F. W.; J. Chem. Phys. 1980, 73, 2871.

18. Bader, R. F. W.; Monatshefte füur Chemie 2005, 136, 819.

19. Drude, P.; Annalen der Physik 1900, 1, 566.

20. Drude, P.; Annalen der Physik 1900, 3, 369.

21. Morgon, N. H.; Custodio, R.; Quim. Nova. 1995, 18, 44.

22. Geerlings, P.; De Proft, F.; Langenaeker, W.; Chem. Rev. 2003, 103, 1793.

23. Hohenberg, P.; Kohn, W.; Phys. Rev. B 1964, 136, 864.

24. Kohn, W.; Sham, L. J.; Phys. Rev. A 1965, 140, 1133

25. Parr, R. G.; Ayers, P. W.; Nalewajski, R. F.; J. Phys. Chem. A 2005, 109, 3957.

26. Matta, C. F.; Bader, R. F. W.; J. Phys. Chem. A 2006, 110, 6365.

27. Popelier, P. L. A.; Coord. Chem. Rev. 2000, 197, 169.

28. Bader, R. F. W.; Acc. Chem. Res. 1985, 18, 9.

29. Nasertayoob, P.; Shahbazian, S.; J. Mol. Struct. (THEOCHEM) 2008, $869,53$.

30. Keith, T. A.; Bader, R. F. W.; Aray, Y.; Int. J. Quantum Chem. 1996, 57, 183.

31. Mohallem, J. R.; Theor. Chem. Acc. 2002, 107, 372.

32. Popelier, P. L. A.; Aicken, F. M.; J. Am. Chem. Soc. 2003, 125, 1284.

33. Hati, S.; Datta, D.; J. Comput. Chem. 1992, 13, 912.

34. Mulliken, R. S.; J. Chem. Phys. 1955, 23, 1833.

35. Araújo, R. C. M. U.; da Silva, J. B. P.; Ramos, M. N.; J. Mol. Struct. (THEOCHEM) 1995, 51, 821.

36. O`Brien, S. E.; Popelier, P. L. A.; Can. J. Chem. 1999, 77, 28.

37. Popelier, P. L. A.; J. Phys. Chem. A 1999, 103, 2883.

38. Bader, R. F. W.; J. Phys. Chem. A 1998, 102, 7314.

39. Frenking, G.; Esterhuysen, C.; Kovacs, A.; Chem. Eur. J. 2006, 12, 7773.

40. Alcoba, D. R.; Lain, L.; Torre, A.; Bochicchio, R. C.; Chem. Phys. Lett. 2006, 426,426

41. Marcin, P.; Grabowski, S. J.; J. Chem. Res. 2004, 7, 492.

42. Rode, J. E.; Dobrowolski, J. Cz.; Chem. Phys. Lett. 2007, 449, 240.

43. Alsberg, B. K.; Marchand-Geneste, N.; King, R. D.; Chemom. Intell. Lab. Syst. 2000, 54, 75.

44. Fradera, X.; Austen, M.A .; Bader, R. F. W.; J. Phys. Chem. A 1999, 103, 304.

45. Grabowski, S. J.; Sokalski, W. A.; Dyguda, E.; Leszczy ski, J.; J. Phys. Chem. B 2006, 110, 6444

46. Bader, R. F. W.; Nguyen-Dang, T. T.; Tal, Y.; Rep. Prog. Phys. 1981, 44, 893.

47. Bader, R. F. W.; Beddall, P. M.; J. Chem. Phys. 1972, 56, 3320.

48. Bader, R. F. W.; Pure Appl. Chem. 1988, 60, 145.

49. Vener, M. V.; Manaev, A. V.; Egorova, A. N.; Tsirelson, V. G.; J. Phys. Chem. A 2007, 111, 1155; Hugas, D.; Simon, S.; Duran, M.; Struct. Chem. 2005, 16, 257; Oliveira, B. G.; Vasconcellos, M. L. A. A.; J. Mol. 
Struct. (THEOCHEM) 2007, 774, 83; Oliveira, B. G.; J. Chil. Chem. Soc. 2009, 54, 43; Oliveira, B. G.; Vasconcellos, M. L. A. A.; Acta. Chim. Slov. 2009, 56, 340; Oliveira, B. G.; J. Arg. Chem. Soc. 2007, 95, 59; Oliveira, B. G.; J. Arg. Chem. Soc. 2008, 96, 42; Oliveira, B. G.; Vasconcellos, M. L. A. A.; Olinda, R. R.; Filho, E. B. A.; Struct. Chem. 2009, $20,81$.

50. Filho, E. B. A.; Ventura, E.; do Monte, S. A.; Oliveira, B. G.; Junior, C. G. L.; Rocha, G. B.; Vasconcellos, M. L. A. A.; Chem. Phys. Lett. 2007, $449,336$.

51. Keith, T. A.; The Quantum Theory of Atoms in Molecules: From Solid State to DNA and Drug Design, Wiley-VCH, Weinheim, 2007.

52. Bader, R. F. W.; Hernández-Trujillo, J.; Cortés-Guzmán, F.; J. Comput. Chem. 2007, 28, 4.

53. Gillespie, R. J.; Molecular Geometry, van Nostrand-Reinhold: London, 1978.

54. Popelier, P. L. A.; Sci. Comput. World 1999, 45, 26.

55. Koch, U.; Popelier, P. L. A.; J. Phys. Chem. 1995, 99, 9747.

56. Bader, R. F. W.; Fang, D.-C.; J. Chem. Theor. Comput. 2005, 1, 403; Sobczyk, L.; Grabowski, S. J.; Krygowski, T. M.; Chem. Rev. 2005, 105, 3513; Oliveira, B. G.; Araújo, R. C. M. U.; Carvalho, A. B.; Ramos, M. N.; J. Mol. Model. 2009, 15, 123.

57. Matta, C. F.; Boyd, R. J.; The Quantum Theory of Atoms in Molecules, Wiley-VCH Verlag \& Co. KgaA: Weinheim, 2007.

58. Zhang, Y.-H.; Hao, J.-K.; Wang, X.; Zhou, W.; Tang, T.-H.; J. Mol. Struct. (THEOCHEM) 1998, 455, 85.

59. Rozas, I.; Alkorta, I.; Elguero, J.; J. Phys. Chem. A 1997, 101, 9457.

60. Oliveira, B. G.; Araújo, R. C. M. U.; Pereira, F. S.; Lima, E. F.; Silva, W. L. V.; Carvalho, A. B.; Ramos, M. N.; Quim. Nova 2008, 31, 1673.

61. Oliveira, B. G.; Araújo, R. C. M. U.; Carvalho, A. B.; Lima, E. F.; Silva, W. L. V.; Ramos, M. N.; Tavares, A. M.; J. Mol. Struct. (THEOCHEM) 2006, 775, 39.

62. Bobadova-Parvanova, P.; Boris Galabov, B.; J. Phys. Chem. A 1998, 102, 1815 .

63. Oliveira, B. G.; Pereira, F. S.; Araújo, R. C. M. U.; Ramos, M. N.; Chem. Phys. Lett. 2006, 427, 181.

64. Oliveira, B. G.; Araújo, R. C. M. U.; Carvalho, A. B.; Ramos, M. N.; Chem. Phys. Lett. 2007, 433, 390.

65. Oliveira, B. G.; Araújo, R. C. M. U.; Carvalho, A. B.; Ramos, M. N.; J. Theor. Comp. Chem. 2007, 6, 647.

66. Oliveira, B. G.; Araújo, R. C. M. U.; Carvalho, A. B.; Ramos, M. N.; Quim. Nova 2007, 30, 1167.

67. Oliveira, B. G.; Araújo, R. C. M. U.; Carvalho, A. B.; Ramos, M. N.; Orbital-Elec. J. Chem. 2009, 1, 167.

68. van Duijneveldt, F. B.; Murrell, J. N.; J. Chem. Phys. 1967, 46, 1759.

69. Boys, S. B.; Bernardi, F.; Mol. Phys. 1970, 19, 553.

70. McQuarrie, D. A.; Statistical Thermodynamics, Harper and Row: New York, 1973.

71. Castillo, N.; Matta, C. F.; Boyd, R. J.; Chem. Phys. Lett. 2005, 409, 265.

72. Alkorta, I.; Barrios, L.; Rozas, I.; Elguero, J.; J. Mol. Struct. (THEOCHEM) 2000, 496, 131

73. López, C. S.; Faza, O. N.; Cossío, F. P.; York, D. M.; de Lera, A. R.; Chem. Eur. J. 2005, 11, 1734.
74. Oliveira, B. G.; Araújo, R. C. M. U.; Chagas, F. F.; Carvalho, A. B.; Ramos, M. N.; J. Mol. Model. 2008, 14, 949.

75. Grabowski, S. J.; Hydrogen Bonding - New Insights, Springer: Amsterdam, 2006

76. Martin, T. W.; Derewenda, Z. S.; Nat. Struct. Bio. 1999, 6, 403.

77. Richardson, T.; de Gala, S.; Crabtree, R. H.; Siegbahn, P. E. M.; J. Am. Chem. Soc. 1995, 117, 12875.

78. Grabowski, S. J.; Leszczynski, J.; Practical Aspects of Computational Chemistry, Springer: Amsterdam, 2009.

79. Grabowski, S. J.; Sokalski, W. A.; Leszczynski, J.; J. Phys. Chem. A 2004, 108, 5823 .

80. Oliveira, B. G.; Araújo, R. C. M. U.; Ramos, M. N.; Struct. Chem. 2008 , 19,185 .

81. Bakhmutov, V. I.; Dihydrogen Bond: Principles, Experiments, and Applications, Wiley-VCH \& Sons: New York, 2008.

82. Custelcean, R.; Jackson, J. E.; Chem. Rev. 2001, 101, 1963.

83. Oliveira, B. G.; Araújo, R. C. M. U.; Ramos, M. N.; Struct. Chem. 2008, 19,665 .

84. Oliveira, B. G.; Vasconcellos, M. L. A. A.; Struct. Chem. 2009, 20, 897.

85. Grabowski, S. J.; J. Mol. Struct. 2000, 553, 151.

86. Oliveira, B. G.; Araújo, R. C. M. U.; Ramos, M.N.; Struct. Chem. 2008 , 19,185 .

87. Oliveira, B. G.; Araújo, R. C. M. U.; Quim. Nova 2007, 30, 791

88. Yang, Y.; Int. J. Quantum Chem. 2009, 109, 266.

89. Pinchas, S.; Anal. Chem. 1955, 27, 2.

90. Trudeau, G.; Dumas, J. M.; Dupuis, P.; Guérin, M.; Sandorfy, C.; Top. Curr. Chem. 1980, 93, 91.

91. Hobza, P.; Špirko, V.; Havlas, Z.; Buchhold, K.; Reimann, B.; Barth, H.-D.; Brutschy, B.; Chem. Phys. Lett. 1999, 229, 180.

92. Hobza, P.; Havlas, Z.; Chem. Rev. 2000, 100, 4253.

93. Oliveira, B. G.; Ramos, M. N.; Int. J. Quantum Chem. 2010, 110, 307, doi: 10.1002/qua.21995.

94. Grabowski, S. J.; Sokalski, W. A.; Leszczynski, J.; Chem. Phys. 2007, $337,68$.

95. Oliveira, B. G.; Leite, L. F. C. C.; J. Mol. Struct. (THEOCHEM) 2009 , 915, 38 .

96. Oliveira, B. G.; Vasconcellos, M. L. A. A.; Inorg. Chem. Comm. 2009 $12,1142$.

97. Yu, W.; Lin, Z.; Huang, Z.; Chem. Phys. Chem. 2006, 7, 828.

98. Jansen, L.; Phys. Rev. 1967, 162, 63.

99. Bader, R. F. W.; Matta, D. F.; J. Phys. Chem. A 2004, 108, 8385.

100. Matta, C. F.; Popelier, P. L. A.; An Introduction to the Quantum Theory of Atoms in Molecules, Wiley-VCH Verlag GmbH \& Co. KgaA: Weinheim, 2007

101. Bader, R. F. W.; An Introduction to the Electronic Structure of Atoms and Molecules, Clarke, Irwin \& Co. Ltd.: Toronto, 1970

102. Chaudry, U. A.; Popelier, P. L. A.; J. Org. Chem. 2004, 69, 233; Bader, R. F. W.; Chem. Phys. Lett. 2006, 426, 226; Otero, N.; Mandado, M.; Mosquera, R. A.; J. Chem. Phys. 2007, 126, 234108; Rodríguez, J. I.; Ayers, P. W.; Götz, A. W.; Castillo-Alvarado, F. L.; J. Chem. Phys. 2009, 131,021101

103. Nasertayoob, P.; Shahbazian, S.; Int. J. Quantum Chem. 2008, 108 , 1477 